\title{
Policy-driven Model for Security Management
}

\author{
K. Boudaoud ${ }^{1}$ and C. McCathieNevile ${ }^{2}$ \\ ${ }^{1}$ CUI- University of Geneva, Switzerland/ University of Nice Sophia Antipolis, France \\ ${ }^{2}$ W3C - INRIA, Sophia Antipolis, France
}

\begin{abstract}
In improving security for the networks of today and tomorrow there are two important features that must be considered. The first is the open and distributed nature of today's enterprise networks. And the second is their dynamic nature, not just in terms of size, but in terms of changing services offered, and the mobility of users. Of particular importance, then is the ability to rapidly apply new security policies in a system. Equally, a system should incorporate as much "intelligence" and reactivity in the system as possible. Many existing security solutions are designed for a static, known network, and are therefore unsuited to the requirements of today's enterprise networks. In order to provide a system that can readily handle these requirements, recent works have shown that multi-agents systems offer a promising platform. The focus of our work concerns one critical security management issue that is intrusion detection. In this paper, we propose a model of managing security policies in a multi-agents system for intrusion detection.
\end{abstract}

Keywords: Security management, security policies, intrusion detection, multi-agents system

\section{Introduction}

To improve security of networks, we must deal with two important characteristics, which are their distributed nature and variation in terms of services offered, used resources and number of users. The high level of dynamics in networks requires modifying, at any time, security management functions in order to adapt them to changes that occur in monitored networks. Actually, security policies which are applied in an organization may change. So, the administrator should change or add new ones in order to modify and readapt security management and monitoring functions. Therefore, security solutions to be developed must take into account these variations without having to be restarted. The existing solutions have been designed for well-defined networks and systems. Thus, they are not adapted to dynamical environments, where flexibility and adaptability are primordial criteria, nor to the increasing complexity of user behaviors. Particularly some recent aspects like the mobility of users, enhance this complexity. The mobility imply new security problems such as new vulnerabilities and new kinds of intrusions that must inevitably be considered. This mobility aspect increases the need of having flexible and adaptable security solutions in order to add new capabilities and reconfigure them easily. These characteristics have been the subject of many works, particularly in the network management domain [1]. Recent solutions [1][2] have shown that multi-agent system (MAS)-based approaches are well-suited to resolve complex problems. Therefore, in order to provide security solution for current networks as well as for the next generation, we apply the MAS paradigm to the area of security and propose a new generation of security management architecture based on a MAS to model and implement an intelligent security system.

To manage the security of a network, we need to: 1) manage security policies specified by the administrator, and 2) analyze security events which can characterize security attacks occurring in the network. Based on these elements, we have

The original version of this chapter was revised: The copyright line was incorrect. This has been corrected. The Erratum to this chapter is available at DOI: 10.1007/978-0-387-35620-4_43 
decomposed our intelligent agent-based model of security management in three plans: the user plan, the intelligence plan and the kernel plan [3].

The user plan represents the security policy-based model. It involves the administrator and the security policies. The administrator specifies security policies to apply to the network. He modifies them when the network configuration changes or when he would like to detect new attacks. Moreover, he receives security reports.

The intelligence plan represents the intelligent agent-based model, which involves the MAS and the BDI-based information model [4] used by it. In this information model, we distinguish, three information elements: beliefs, suspicions and goals. This model is described in [3] [5].

The kernel plan represents the event-based model. It consists of the network to secure and the security events occurring in it. These events are analyzed to detect attacks and to make sure that security policies are respected. In this plan is also defined the notion of attack schema [3]. An attack schema is a class that characterizes a kind of security attack. An instance of attack schema is a description of a parameterized attack.

In this paper, we focus on our model of security policies.

This paper is organized as follows. First, we outline the role of security policies in intrusion detection. Then, we describe our model of security policies. Afterwards, we illustrate with an example the derivation of security policies in attack schemas. Finally we conclude with some remarks and foreshadow future works.

\section{Role of security policies in intrusion detection}

Security policies play an important role in our model because they guide the behavior of our MAS. They act at three levels (see Figure 1) and allow to:

- select and instantiate security attack schemas to detect,

- create goals, for relevant agents, in order to detect these security attack schemas,

- select events to filter for recognizing instantiated security attack schemas.

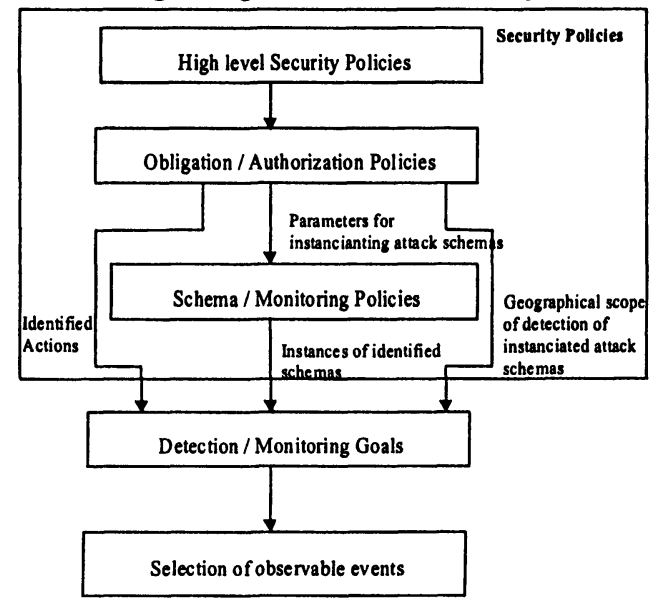

Figure 1. The security policy role

Therefore, security policies, permit, at a lowest level, selection of events to observe. When the administrator specifies security policies, a set of detection goals to reach, are derived from these policies. These goals are created with the instances of attack schemas to detect. From these attack schemas the events classes to collect from the 
network are selected. Thus, when an event occurs in the network, the agent tests if it matches these event classes. If it matches, it is collected.

From the collected events the beliefs of the agent are then created and updated. These beliefs are then analyzed to test if they match an attack schema that has been specified in or by a policy. If it matches, then a detection goal is reached. The beliefs and suspicions of the other agents are also considered when the beliefs are analyzed. When a goal is reached a list of actions (such as informing the administrator,...) are executed.

\section{Management model of security policies}

In this section, we describe the user plan of our model of security management. This plan implements security policies that translate the security rules and procedures of an enterprise network.

In order to be interpreted by the MAS, security policies must be formalized by identifying the different abstraction levels and specifying them in a formal language. Based on existing works [6][7][8], we have proposed a new model for a hierarchy of security policy, where we have identified three abstraction (see Figure 1):

1. the high level security policies that specify the general enterprise security rules,

2. the obligation/authorization policies that specify the obligation and authorization rules. In this level of abstraction we define:

- the different entities (domains to protect, internal or external users) to which these rules are applied.

- the authorized/denied actions and the temporal/geographical constraints.

3. the schema policies that specify the attack schemas to detect. We focus on this policy level because they represent the policies that are finally implemented in our system. In the same level, we have the monitoring policies that specify the monitoring tasks. They permit the administrator to monitor specific activities concerning a part of the network or a particular user. Concerning the schema policies, we define: schema instantiation policies and response to attack schemas policies.

The schema instantiation policies define attack schemas. The instantiation of attack schema is not always done in the same manner, but depends on whether the superior level policy is an obligation or authorization one.

The authorization policies permit the administrator to define and to create new attack schemas. An authorization policy could be positive or negative, and the process for each is different. From a positive authorization, we must deduce the attack schemas denying the authorized actions. From a negative authorization, we deduce the attack schemas defined directly in the authorization policy.

The obligation policies enable the system to detect specific attacks by instantiating existing schemas in order to specify what we really want to deny. The instantiation of a schema is done either: nominatively (i.e. with regard to a user, a particular source network,...) or anonymously without specifying the source and/or destination. Moreover, the detection zone of an attack can be delimited by the administrator, to either: the whole enterprise network or a part of the network, when he wants to protect strongly this zone. Thus, the obligation policy acts on either: the instance itself of an attack schema or on the schema instance and the geographical detection zone.

The response to attack schemas policies, which are specified by obligation policies in the superior level, allow the system to implement system reactions when an attack is detected. They define the actions that the administrator and the agents must 
do or not in this case such as: closing or not a connection, filtering source or destination addresses by modifying the parameters of a filter or a firewall.

A detailed description of the formalism used for specifying a policy is given in [3].

4. Some examples

4.1. Example 1: Creation of an attack schema by deriving a negative authorization policy

In this example, we want to create an attack schema from a negative authorization policy.

The High level policy

The high level policy is to: "deny repeated failed connections coming from any source and going to any destination"

The Authorization policy

The authorization policy $P_{1}$ will be defined by the following parameters:

PolicyId

P01

PolicyBehavior

detection of repeated login failure coming from the Site A

Policy Owner
Policy Type and going to the Web server of the local network $B$.

Mode Karima Boudaoud detection policy

negative authorization

Life Time

Status

Attack Name

Events List

Subject

Target

Detection Domain

from 10-10-2000 to 31-12-2000

activated

Doorknob Rattling

e

same

anyone

Constraints

indifferent

window observation $=120 \mathrm{sec}$

e. ObservationPoint $=$ network traffic

e.EventType $=$ connection

e. EventName $=$ telnet

e.Event Source $=$ same

e.EventDestination = anyone

e.EventResult $=$ failure

e.Activity = extranet

operators.frequency $(e, 5)$

Date

10-10-2000

The schema policy

From the authorization policy, we obtain a schema policy, which is defined by : the "schemaPolicyId" and the attack schema created. So, the attack schema created is named "Doorknob Rattling" and is represented, in a simplified version, by the following parameters:

Schema Name

Window Observation

Doorknob Rattling

Event Description List

120 seconds

e (Observation Point

Event Type

Event Name

network traffic

Event Source

connection

telnet

same 


$\begin{array}{cl}\text { Event Destination } & \text { anyone } \\ \text { Event Result } & \text { failure } \\ \text { Activity } & \text { extranet ) } \\ \text { Series Attribute Operator List (Operator Type } & \text { Iteration } \\ \text { Event Description } & \text { e } \\ \text { Frequency } & 5 \text { ) }\end{array}$

\subsection{Example 2: Instantiation of an attack schema by deriving an} obligation policy

In this example, we want to instantiate, from an obligation policy, the schema created previously.

The High level policy

The high level policy is to: "deny repeated failed connections to the local area network A and coming from Site $Z "$

The Obligation policy

The obligation policy $P_{2}$ will be defined by the following parameters: PolicyId $\mathrm{P} 02$

PolicyBehavior detection of repeated login failure coming from the Site A and going to the Web server of the local network $\mathrm{B}$.

Policy Owner Karima Boudaoud

Policy Type from 10-10-2000 to31-12-2000

Status

Attack Name activated

Subject

Doorknob Rattling

Target

SITE A

Detection Domain

Web Server

Actions

Local Network B

Date inform administrator 10-10-2000

In this policy, we find:

- the zone of detection, which is Local Network B,

- the attack schema class, which is Doorknob Rattling $\left(S_{c h 1}\right)$,

- the schema parameters to instantiate which are: 1) the source equal to SITE $A$ and 2) the destination equal to Web Server $C$,

- the actions to execute when an attack is detected which is to inform the administrator.

\section{The schema policy}

From the obligation policy, we obtain a schema policy, which is defined by its "schemaPolicyId" and the instantiated attack schema "Doorknob Rattling", created previously. This attack schema is instatiated with the parameters specified in the obligation policy.

Schema Name

Window Observation

Event Description List

\section{Doorknob Rattling}

120 seconds

e (Observation Point

Event Type

Event Name

Event Source

Event Destination network traffic

connection

telnet

SITE A

local network B.Web server 


$\begin{array}{cl}\text { Event Result } & \text { failure } \\ \text { Activity } & \text { extranet) } \\ \text { Series Attribute Operator List (Operator Type } & \text { Iteration } \\ \text { Event Description } & \text { e } \\ \text { Frequency } & 5 \text { ) }\end{array}$

\section{Distribution of attack schemas}

This instance of attack schema is then translated into the form of detection goals, which are distributed and delegated to the different agents monitoring the Local Network $B$. When the agents receive these goals, they update the set of event classes to filter with telnet events. So, they collect all the telnet events coming from the SITE $A$ and going to the $W e b$ server $C$. The different agents analyze then the filtered events and if the frequency 6 is reached by one agent, the administrator is informed by the this agent. A detailed version of distribution and detection of attacks is given in [3].

\section{Conclusion}

In this paper, we presented the main components of our model of security management: the security policies. In the introduced model, security policies essentially instantiate attack schemas to detect. To represent them, we had: 1) on one hand, to study existing models; 2) on the other hand to consider the language of representation of attack schemas that we defined in [3]. Therefore, we proposed a new model, where we identified two main levels of abstraction: 1) a level for representing obligation / authorization policies; 2) a level for representing schema policies. The schema policies allow us to instantiate attack schemas that are then sent, as goals, to the MAS. The goal of the agents is therefore to detect attacks specified by the received attack schemas. This work has been implemented in JAVA with the multi-agent plate-form DIMA [9]. We are now working on the experimental validation of the implemented system. To improve the human interaction interface, we plan to introduce an interface to deduce the formal specification from a natural language description of the management policy.

\section{References}

[1] R. F. Teixeira de Oliveira, "Gestion des Réseaux avec Connaissance des Besoins: Utilisation des Agents Logiciel", PhD. thesis ENST, Paris, 1998.

[2] A. S. Rao and M. P. Georgeff, "Intelligent Real-Time Network Management", Technical report 15, Australian AI Institute, Carlton, Australia, 1991.

[3] K. Boudaoud. "Intrusion Detection: a new approach using a multi-agent system", $\mathrm{PhD}$ thesis, Institut Eurecom/ EPFL, Sophia Antipolis, 2001.

[4] A. S. Rao and M. P. Georgeff, "BDI - agents: from theory to practice", Proc. of the First International Conference on Multi - Agent Systems, San Francisco, 1995.

[5] K. Boudaoud and Z. Guessoum. "A Multi-agents System for Network Security Management". Proc. of the Sixth IFIP Conference on Intelligence in Networks (SmartNet'200), Vienna, Austria, September2000.

[6] S. Heilbronner, "Requirements for Policy-Based Management of Nomadic Computing Infrastructures", Proc. of the Sixth Workshop of the HP-OVUA, Bologna, June 1999.

[7] R. Wies. "Policies in Integrated Network and Systems Management: Methodologies for the Definition, Transformation and Application of Management Policies", 1995

[8] N. Yialelis and M. Sloman, "A Security Framework Supporting Domain Based Access Control in Distributed Systems", Imperial College Research Report Doc 1995/14, Department of Computing, Imperial College of Science Technology and Medicine, 1995.

[9] Z. Guessoum and J.-P. Briot, "From Active Object to Autonomous Agents", IEEE Concurrency, volume $7 \mathrm{~N}^{\circ} 3$, July/September, 1999, pp. 68-78. 